\title{
The Mechanical and Thermal Design for the MICE Detector Solenoid Magnet System*
}

\author{
P. Fabbricatore, S. Farinon, M. Perrella, U. Bravar, and M. A. Green
}

\begin{abstract}
The detector solenoid for MICE surrounds a scintillating fiber tracker that is used to analyze the muon beam within the detector. There are two detector magnets for measuring the beam emittance entering and leaving the cooling channel that forms the central part of the experiment. The field in the region of the fiber detectors must be from 2.8 to $4 \mathrm{~T}$ and uniform to better than 1 percent over a volume that is $300 \mathrm{~mm}$ in diameter by $1000 \mathrm{~mm}$ long. The portion of the detector magnet that is around the uniform field section of the magnet consists of two short end coils and a long center coil. In addition, in the direction of the MICE cooling channel, there are two additional coils that are used to match the muon beam in the cooling channel to the beam required for the detectors. Each detector magnet module, with its five coils, will have a design stored-energy of about $4 \mathrm{MJ}$. Each detector magnet is designed to be cooled using three $1.5 \mathrm{~W}$ coolers. This report presents the mechanical and electrical parameters for the detector magnet system.
\end{abstract}

Index Terms-Superconducting Solenoids, and Muon Cooling

\section{INTRODUCTION}

$\mathrm{T}$ he development of a muon collider or a neutrino factory requires that beams of low emittance muons be produced.

A key to the production of low emittance muons is muon cooling. A demonstration of muon cooling is essential to the development of muon accelerators and storage rings [1], [2]. The international Muon Ionization Cooling Experiment (MICE) will be a demonstration of muon cooling in a configuration of superconducting magnets [3] that may be useful for a neutrino factory.

Ionization cooling of muons means that muons have their momentum reduced in both the longitudinal direction and the transverse direction by passing them through a low $\mathrm{Z}$ absorber. RF cavities are used to re-accelerate the muons to their original momentum. If the scattering in the absorbing medium is not too large, the reaccelerated muon beam will have a lower emittance than the muon beam that entered the absorbers. In order to reduce the multiple scattering of the muon beam in the absorber, the muon beam beta must be low in the absorber and the absorber must have a low $\mathrm{Z}$. The candidate absorbers include hydrogen (either as liquid or gas), lithium hydride, lithium metal or beryllium metal.

Manuscript received 5 October 2004. P. Fabricatore, and S. Farinon, are from INFN Genoa, Genoa Italy. M. Perrella is from Ansaldo Superconduttori Corso Perrone, Genoa Italy. U. Bravar and M. A. Green (email: MAGreen@lbl.gov) are from the Oxford University Physics Department, Oxford OX1-3RH. *This work was supported by the Oxford University physics Department and the Particle Physics and Astronomy Research Council of the United Kingdom.

\section{The Proposed MICE EXPERIMENT}

The proposed MICE experiment will test cooling on a low intensity muon beam from the ISIS ring at the Rutherford Appleton Laboratory in the United Kingdom. The pions that will decay into muons will be produced by dipping a metal target into the ISIS proton beam. The Pions will decay into a well-defined muon beam. These muons will be collected and they will be carried to an experimental hall containing the MICE by a beam transport system.

The beam enters the experiment by passing through a foil that will scatter the muons to produce a beam of desired emittance. The muon emittance will be measured by four planes of scintillating fibers that are in a uniform solenoidal magnetic field from 2.8 to $4 \mathrm{~T}$. Fig. 1 shows schematic a cross-section view of MICE. Fig. 1 shows the cooling channel and the two detectors at both ends of the experiment.

Once the emittance of the muon beam entering the cooling section has been measured, the beam passes through an absorber that cools the muon beam by ionization cooling. The absorber is in a high magnetic region where the field either goes through zero to the opposite polarity or is reasonably uniform (depending on which cooling mode is being tested). In either case, the beam beta is supposed to be minimized in the absorber. The MICE test absorbers may be liquid hydrogen, liquid helium, beryllium, or plastic. The preferred cooling absorber material is hydrogen, because it has the lowest multiple scattering of any of the absorber materials for a given energy absorption and beam beta. The absorber is surrounded by a two-coil solenoid that produces a uniform field or a cusp field that flips as one goes along the axis [4].

The muon beam momentum is recovered by accelerating the beam with a four cell $201.25 \mathrm{MHz}$ RF cavities that are within the coupling magnet 2.0 to $2.5 \mathrm{~T}$ field [5]. After the muon beam has been re-accelerated, it passes through a second absorber in a second low beta region. The process of re-acceleration is repeated then the beam passes through the final absorber. At this point, the beam should be cooled enough, so that the cooling can be clearly measured.

Once the beam has passed through the ionization cooling section, it enters the second detector section. The second detector section is identical to the first detector, except for the time of flight (TOF) detectors at the ends of the experiment. A critical problem for the MICE experiment is matching between the uniform field in the detector region to the rest of the cooling channel where the magnetic field is anything but uniform. An important part of the design of the MICE detector solenoid is the beam beta matching coils between the detector and the cooling channel. 


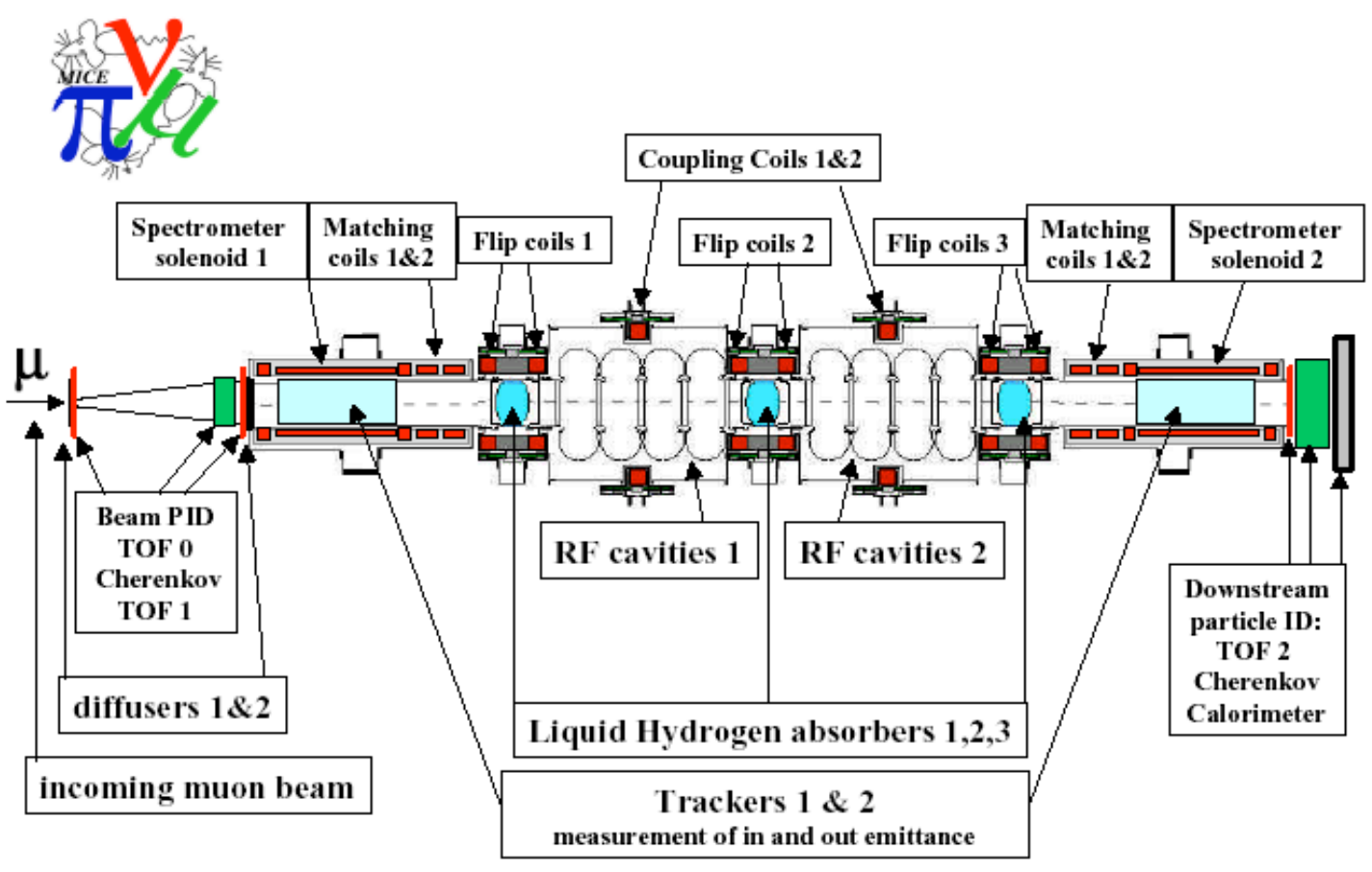

Fig. 1. A Schematic Representation of the MICE Experiment shown in Cross-section. The detector magnet is a combination of matching the coils plus a spectrometer solenoid that is around the tracker. Not shown are the iron shields at the detector magnet ends.

\section{The Detector Module For MICE}

The detector module consists of a radiation shield, a magnet matching section, the uniform magnetic field section, and an iron plate that shields the photo multiplier tubes (PMTs) from the detector magnet stray field. The PMTs are part of the TOF detector that is located $400 \mathrm{~mm}$ from the end coil of the magnet module. Figure 2 shows the detector module with its major components.

At the absorber focus coil end of the detector module is a radiation shield. The radiation shield, which is like a vacuum gate valve, is the equivalent to $50-\mathrm{mm}$. of lead (or say $30 \mathrm{~mm}$ of tungsten), The shield is used to protect the scintillating fiber detectors that are located in the uniform field region of the detector solenoid, while the RF cavities are being conditioned to run at high acceleration gradients. During the cavity conditioning process, the RF cavities produce electrons and $\mathrm{x}$-rays that can cause the plastic fibers to turn brown. During the time the RF cavities are being conditioned, there will be no liquid hydrogen in the absorber that is between the cavities and the detector module. The absorbers will stop radiation produced by the cavities, during the normal operation of MICE.

The iron shield at the other end of the detector magnet module (the end away from the cooling channel) is used to shield the PMT. This iron shield is about $1500-\mathrm{mm}$ in diameter, with a hole around the axis of the experiment that is $500-\mathrm{mm}$ in diameter. The shield thickness is $100 \mathrm{~mm}$. Because the position of the PMT with respect to the time of flight detector is very important, the shield must be close to the end of the detector solenoid. Based on an optimization study, the distance from the end of the detector solenoid to the iron was set at $400 \mathrm{~mm}$. The iron shield affects the detector solenoid by causing a field error in the uniform field section of the detector solenoid. Fortunately, this field error can be corrected with a change in the current in the end coil closest to the shield by up to 2.5 percent. The shield, which is at room temperature, causes a longitudinal force of $100 \mathrm{kN}$ (about 10 tons) toward the iron. This force counters the force due to the field that goes around the end of the solenoid, thus reducing the total longitudinal force on the detector solenoid cold mass supports.

The magnet between the radiation shield and the iron shield consists of two parts. There is the uniform field detector solenoid that consists of a single $1260-\mathrm{mm}$ long center coil with one $120-\mathrm{mm}$ long end coil at each end. The second section of the detector solenoid consists of a pair of 202-mm long coils that matches the muon beam from the focusing magnet of the MICE cooling channel to the threecoil detector solenoid. The five coils are hooked together at $4.2 \mathrm{~K}$ so that the longitudinal magnetic forces can be carried along the solenoid. The largest inter-coil longitudinal force is about $1370 \mathrm{kN}$ (140 tons) from the end coil near the iron shield to the center coil of the detector set. It is proposed that the three detector coils be hooked in series and that each of the match coils have its own set of leads. A pair of low current leads across the center coil of the detector center coil permits each of the end coils to be separately tuned so that the effect of the iron shield can be minimized and so that the tuning process between the focusing module and the detector module can be optimized. The detector magnet warm bore is $400 \mathrm{~mm}$ in diameter. The outer diameter of the magnet is about $1000 \mathrm{~mm}$. The length of the module is $3359 \mathrm{~mm}$. The detector magnet parameters are shown in Table 1. 


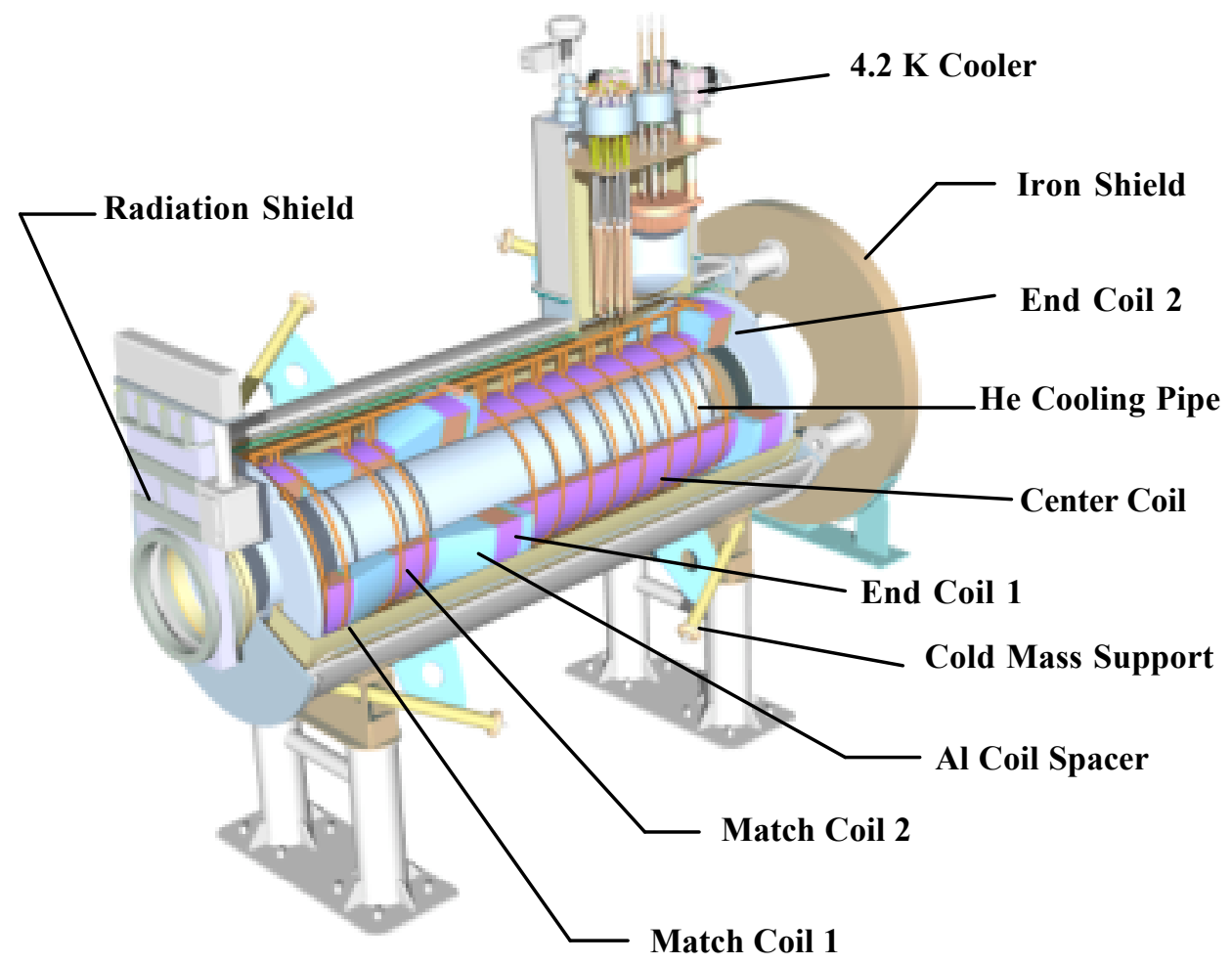

Fig. 2. A 3 Dimensional View of the MICE Detector Solenoid Module that can produce a Uniform Magnetic Field 1-meter Long Region that is 0.3 meters in Diameter. The module also includes the $1.5 \mathrm{~W}$ coolers, the iron shield and the radiation shield.

TABLE 1. THE NOMINAL DESIGN PARMAETERS FOR THE MICE DETECTOR COLENOID COILS

\begin{tabular}{|c|c|c|c|c|c|}
\hline Parameter & Match 1 & Match 2 & End 1 & Center & End 2 \\
\hline $\mathrm{Z}$ distance from MICE Center* $(\mathrm{mm})$ & 3510 & 3951 & 4391 & 4571 & 5891 \\
\hline Coil Length (mm) & 202 & 202 & 120 & 1260 & 120 \\
\hline Coil Inner Radius (mm) & 255 & 255 & 255 & 255 & 255 \\
\hline Coil Thickness (mm) & 100 & 57 & 138 & 50 & 149 \\
\hline Number of Layers & 60 & 34 & 82 & 30 & 90 \\
\hline Number of Turns per Layer & 84 & 84 & 50 & 525 & 50 \\
\hline Coil Overall Current Density $\left(\mathrm{A} \mathrm{mm}^{-2}\right)$ & 66.82 & 73.25 & 61.59 & 64.44 & 67.11 \\
\hline Coil Current (A) & 267.8 & 293.8 & 248.8 & 257.8 & 266.7 \\
\hline Coil Self Inductance (H) & 15.9 & 5.0 & 12.6 & 47.0 & 15.1 \\
\hline Peak Induction in the Coil (T) & 4.8 & 3.8 & 4.9 & 4.0 & 5.2 \\
\hline Coil Stored Energy at Current above (MJ) & 0.57 & 0.22 & 0.39 & 1.56 & 0.54 \\
\hline
\end{tabular}

\section{The Detector Magnet CoIl Design}

The design of the magnet coils shown in Table 1 is based on copper based $\mathrm{Nb}$-Ti conductor that has bare dimensions of $1.52 \mathrm{~mm}$ by $2.27 \mathrm{~mm}$. The insulated dimensions are about $1.65 \mathrm{~mm}$ by $2.40 \mathrm{~mm}$. The number of layers in each coil is even because one wants the coil leads come out at the same end and one wants to minimize the effects of the winding pitch on the field quality.

The three spectrometer coils are hooked in series and are connected to a pair of 300 A leads. The two end coils can be tuned through leads that are connected to the ends of the center coil. The maximum tuning needed is about $20 \mathrm{~A}$.
The self-inductance of the three coils in series is $92 \mathrm{H}$. The spectrometer magnet stored energy at $258 \mathrm{~A}$ is $3.06 \mathrm{MJ}$. The stored energy of the spectrometer magnet is high enough to worry about quench protection. The matrix current density is $80 \mathrm{~A} \mathrm{~mm}^{-2}$, so the $\mathrm{Ej}^{2}$ limit is about $2.3 \times 10^{22} \mathrm{~J} \mathrm{~A}^{2} \mathrm{~m}^{-4}$. The quench protection of the spectrometer coils can be by quench back from a mandrel, or by a resistor put across the leads.

The two match coils are each powered separately through a pair of $300 \mathrm{~A}$ current leads. The full current leads are on these coils, because the current can vary from 50 A to 293 A. From the standpoint of a magnet quench the stored energy of these coils is quite low, so the coils are self-protected without a quench protection system. 
The design of the detector magnet is conservative. The stress within the coils and the aluminum spacers is well below the yield stress of the materials in the coil. Since the detector magnet design is quite conservative, the temperature margin for all of the coils in the magnet is quite high $(>1.7$ $\mathrm{K}$ at $4.2 \mathrm{~K}$ ). The extra temperature margin is a blessing should the heat leak into the magnet be larger than expected.

An aluminum spacer between the coils carries the longitudinal forces that result from the magnet excitation and its interaction with the near by focusing magnet and iron shield. The largest longitudinal force within the detector magnet is about $1370 \mathrm{kN}$ ( $\sim 140$ metric tons). The direction of this force is toward the other coils in the magnet. This force is between end coil 2 and the center coil. The longitudinal force on the center coil is quite low, because the field is uniform along the axis within that magnet. The two match coils and end coil 1 generate longitudinal forces that are in the direction that counters the $1370 \mathrm{kN}$ force on end coil 2. The net force on the detector magnet cold mass support under the highest operating field for MICE is expected to be about $320 \mathrm{kN}$ (about 33 tons). When one considers the peak forces that may occur during a quench, the cold mass support system should be designed for a force of 400 to $500 \mathrm{kN}$ (about 40 to 50 metric tons). A support system based on oriented fiber fiberglass epoxy tension bands can both carry the design longitudinal force and have a low enough heat leak to permit the magnet to be cooled using small coolers.

\section{Cooling The Magnet with Small Coolers}

All of the magnets in MICE will be cooled using small coolers. It is planned that the detector magnet will have three coolers on it. The system shown in Figure 2 has two $1.5 \mathrm{~W}$ coolers at $4.2 \mathrm{~K}$ plus a third cooler operating at about $40 \mathrm{~K}$. Studies at Oxford University [6] suggest that the detector magnet can be cooled using a pair of $1.5 \mathrm{~W}$ coolers, but one might eat into the magnet temperature margin in the event of a fabrication error that results in a higher heat leak into the magnet than it is design. Using three $1.5 \mathrm{~W} 4.2 \mathrm{~K}$ coolers will yield the lowest operating temperatures for the magnet.

Because one is using small coolers to cool both the shield and the magnet, the leads between the shield temperature and $4.2 \mathrm{~K}$ must be HTS leads. Even with HTS leads between the first and second stages of the cooler the current lead heat leak into the magnet is one of the dominant heat leaks.

The largest heat leak into the first stage of the cooler is from the copper leads that carry current into the magnet from room temperature. (This heat leas is about $75 \mathrm{~W}$.) The large number of leads into the detector magnet (3 pairs of $300 \mathrm{~A}$ leads plus 1 pair of $60 \mathrm{~A}$ leads) dictate that three coolers will be needed to cool the magnet.
An important issue when using small coolers is the connection of the cooler to the magnet. Because of the length of the magnet, cryogen free operation is not advisable. The temperature drop from the magnet hot spot to the cooler second stage cold heads is too large. As a result, the connection between the magnet and the cooler second stage cold heads will be made through a gravity feed heat pipe. The temperature difference between the magnet hot spot and the second-stage cold heads can be reduced to $<0.2 \mathrm{~K}$. This temperature difference is not dependent on the distance between the cooler cold head and the magnet. There are two factors that do affect the temperature difference between the cold heads and the magnet. They are the surface area of the condenser attached to the cold heads and the surface area in direct contact with the liquid helium in the magnet coils.

\section{CONCLUding COMMENTS}

The superconducting detector solenoids for MICE can be built with existing niobium titanium conductors. There is enough margin in the detector magnet design to allow for tuning of the MICE magnets and still have reasonably large temperature margins for each of the detector coils. The forces within the magnet can he handled by the proposed coil and aluminum spacer structure.

The detector solenoid can be cooled using three coolers. At least two of those coolers must provide cooling at $4.2 \mathrm{~K}$. The third cooler my provide extra cooling at $40 \mathrm{~K}$ to handle the large heat load coming down the copper leads from room temperature. A key factor in using small coolers to cool the detector coils is the temperature difference between the warmest place in the magnet and the second stage cold heads of the coolers. With proper design, the temperature difference can be less than $0.2 \mathrm{~K}$

\section{REFERENCES}

[1] N. Holtkamp and D. Finley Eds., "A Feasibility Study of a Neutrino Source Based on a Muon Storage Ring," FERMI-Pub-00/108E, (2000)

[2] R. B. Palmer, A. Sessler, A. Skrinsky, A. Tollestrup, et al, "Muon Colliders, " Brookhaven National Laboratory Report BNL-62740, January 1996

[3] M. A Green and J. M. Rey, "Superconducting Solenoids for an International Muon Cooling Experiment," IEEE Transactions on Applied Superconductivity 13, No. 2 p 1373 (2003)

[4] S. Q. Yang, M. A. Green, G. Barr et al, "The Mechanical and Thermal Design for the MICE Focusing Solenoid Magnet System," IEEE Transactions on Applied Superconductivity 15, (this volume), (2005)

[5] M. A. Green, S. Q. Yang, U. Bravar, et al, "The Mechanical and Thermal Design for the MICE Coupling Solenoid Magnet," IEEE Transactions on Applied Superconductivity 15, (this volume), (2005)

[6] M. A. Green, "Cooling the MICE Magnets using Small Cryogenic Coolers," an Oxford University report, 10 September 2004. Contact S. Q. Yang of the Oxford University Physics Department (at s.yang@physics.ox.ac.uk) for a copy. 